\title{
Immunoinflammatory responses in gastrointestinal tract injury and recovery
}

\author{
Garima Verma, Akranth Marella, Md. Shaquiquzzaman and Md. Mumtaz Alam $\bowtie$ \\ Department of Pharmaceutical Chemistry, Faculty of Pharmacy, Jamia Hamdard, New Delhi, India
}

Inflammation is a non-specific immune response to infection, irritation or other injury, the key features being redness, warmth, swelling and pain. A number of mediators are released which alter the resistance of mucosa to injury induced by noxious substances. Oxidative stress is a unifying mechanism of injury in many types of disease processes, including gastrointestinal diseases. It has been defined as an imbalance in the activity of pro and antioxidants. Pro-oxidants favour free radical formation while antioxidants inhibit or retard the same. A number of markers of oxidative stress have been identified. This review provides an overview of various mediators of inflammation and oxidative stress, and diverse approaches for prevention and treatment of gastrointestinal inflammation.

Key words: inflammation, infection, oxidative stress

Received: 28 November, 2012; revised: 03 April, 2013; accepted: 03 June, 2013; available on-line: 12 June, 2013

\section{INTRODUCTION}

Inflammation can be defined as the presence of five macroscopic pathological phenomena (proposed by Celsus, as long as 2000 years ago), comprising tumor: swelling of the tissue, calor: elevated tissue temperature, rubor: redness of vascularized tissue at inflammation site, dolor: intensive sensation of a noxious stimulus, and function laesa: impaired functioning of the affected organ (Stankov, 2012). It is an intrinsically beneficial event that leads to the removal of offending factors and restoration of the tissue structure and physiological function (Ricciotti \& Fitz, 2011). The gastrointestinal tract is crucial for the immune system functioning since the majority of immune function $(75 \%)$ takes place there (Rea \& Patel, 2010). The lumen of the gastrointestinal tract is home to an enormous number of bacterial species, and our microbiota generally thrives in a symbiotic relationship with the host (Brenchley \& Douek, 2012).

The gastrointestinal mucosa is exposed to myriads of infectious and toxic agents and food antigens, a unique barrier mechanism handles them on the mucosal surface, and specific immune responses to these antigens challenge the mucosal immune system to maintain homeostasis of the gastrointestinal functions and structure. The function of the gastrointestinal tract in monitoring and sealing the host's interior from intruders is called the gut barrier. A variety of specific and non-specific mechanisms are in operation to establish the barrier. These include luminal mechanisms and digestive enzymes, the epithelial cells together with tight junctions between them, and the gut immune system (DeMeo et al., 2002).
Medilexicon's Medical Dictionary defines inflammation as "A fundamental pathologic process consisting of a dynamic complex of histologically apparent cytologic changes, cellular infiltration, and mediator release that occurs in affected blood vessels and adjacent tissues in response to an injury or abnormal stimulation caused by a physical, chemical or biological agent, including the local reactions and resulting morphological changes, the destruction or removal of the injurious material and the responses that lead to repair and healing."

Inflammation of the mucosal layer of the gastrointestinal tract is not only a feature almost always associated with ulceration of those tissues, but it also plays an important role in both production and healing of lesions (Wallace \& Chin, 1997). It is a homeostatic response aimed at limiting entry of foreign materials to the body and facilitating repair (Martin \& Wallace, 2006). In the gastrointestinal tract the immunoinflammatory process is a key component of the mucosal defense against exogenous and endogenous factors. Impairment of this response can lead to mucosal injury and to an impairment of the repair process. The inflammatory response is coordinated to a large extent by an array of chemical mediators that are released from the epithelium, immunocytes and nerves within lamina propria. This release occurs in response to injury, infection or exposure of cells to antigens (Wallace \& Chin, 1997).

The mucosal defense has been best characterized in the stomach, which exhibits remarkable resistance to the damaging effects of acid and pepsin. It consists of a complex network of components that function in concert with each other. This network includes:

1) Extramucosal components such as acid, mucus, surface active phospholipids and bicarbonate.

2) The epithelium itself.

3) The microcirculation and sensory afferent neurons beneath the epithelium.

4) The mucosal immune system.

5) The ability of the mucosa to undergo repair (Wallace \& Granger, 1996).

The first level of defense consists of factors secreted into the lumen, including acid, mucus, bicarbonate, and antibacterial substances (e.g., immunoglobulins and lactoferrin). The principal function of gastric acid is to reduce the number of ingested bacteria entering the small intestine.

The second level of defense is the epithelium, which is remarkably resistant to acid-induced injury. The epithelium also acts as a barrier to the passive diffusion of

e-mail: drmmalam@gmail.com

Abbreviations: COX, cyclooxygenae; H. pylori, Helicobacter pylori; LXs, lipoxins, NO, nitric oxide; NSAIDs, non-steroidal anti-inflammatory drugs; NO-NSAIDS, nitric oxide-non-steroidal anti-inflammatory drugs; OS, oxidative stress; ROS, reactive oxygen species. 
harmful substances. Damage to the epithelium can be repaired very quickly through a process known as "restitution", which involves migration of healthy epithelial cells from the gastric pits over the denuded region. Restitution is observed in response to injury throughout the GI tract, as well as in other tissues.

The third level of defense is microcirculatory response of mucosa. It is modulated by the extrinsic and intrinsic nervous systems and by diverse inflammatory mediators. Angiogenesis is also recognized as a crucial event in inflammation (Chaitanya et al., 2010). When toxins (including gastric acid) diffuse into the mucosa, there is a profound and rapid increase in the mucosal blood flow. This is mediated via extrinsic primary afferent nerves which release both calcitonin gene related peptide and substance $\mathrm{P}$ in the vicinity of submucosal arterioles resulting in vasodilatation. The increase in blood flow acts to dilute and neutralize the toxin, as well as to prevent the toxins from accumulating within the mucosa in cytotoxic concentrations.

The fourth level of defense is the mucosal immune system, consisting of various immunocytes resident within the lamina propria that act as sentinels. Mast cells and macrophages, for example, can sense the entry of foreign material (e.g., antigens and endotoxin) into the mucosa and can respond by releasing chemical mediators that coordinate an appropriate inflammatory response.

The final level of mucosal defense is called into play when an ulcer has formed - an ulcer being defined as a break in the mucosa that extends through the muscularis mucosae. In these circumstances, the ulcer is repaired through growth and re-development of gastric glands, growth of new blood vessels (angiogenesis), and re-innervation of the mucosa by extrinsic and intrinsic nerves (Wallace \& Chin, 1997).

Various groups of inflammatory mediators contribute to the mucosal defense and repair.

\section{MEDIATORS DERIVED FROM THE ACTION OF CYCLOOXYGENASE: PROSTAGLANDINS}

Prostaglandins are twenty-carbon fatty acid derivatives and peptido-fatty acids derived from membrane phospholipids via the enzyme cyclooxygenase (COX) (Pathmakanthan \& Hawkey, 2000). They generally act in an autocrine and paracrine manner. They mediate various physiological aspects of mucosal defense and the suppression of prostaglandin synthesis in the stomach is a critical event in the development of mucosal injury after non-steroidal anti-inflammatory drug (NSAID) administration (Brzozowski et al., 2008). They modulate many components of mucosal defense. They stimulate mucus and bicarbonate secretion, elevate mucosal blood flow, and increase the resistance of epithelial cells to injury induced by cytotoxins. Prostaglandins can also downregulate the release of a number of other inflammatory mediators that have been suggested to contribute to mucosal injury in certain circumstances.

There are two isoforms of COX: COX-1 and COX2. Only COX-2 plays an important role in mucosal defense. The basal expression of COX-2 is low, but is rapidly increased in response to stimuli.

COX-2-derived prostaglandins make an important contribution to the repair of ulcers. Such repair involves formation of granulation tissue at ulcer base, formation of new blood vessels (angiogenesis) and reestablishment of glandular architecture. COX-2 is strongly expressed in cells at the ulcer margin, which is where epithelial prolif- eration primarily occurs allowing for reestablishment of glands, in the ulcer bed the site of new vessel growth (Martin \& Wallace, 2006).

\section{MEDIATORS DERIVED FROM THE ACTION OF CYCLOOXYGENASE: LIPOXINS (LXS)}

Lipoxins, endogenous eicosanoids, are biosynthesized at inflammation sites (Bonnans et al., 2002). They are oxygenated arachidonic acid trihydoxytetraene-containing derivatives formed during cell to cell interactions and appear to function as endogenous anti-inflammatory mediators. They predominantly counter-regulators of some well-known mediators of inflammation (Neerudu et al., 2009) and act via formyl peptide receptor 2 (Macdonald et al., 2011).

Concurrent administration of aspirin and a selective COX-2 inhibitor results in significantly greater damage than produced by either drug alone. This synergistic interaction has been observed in humans. Aspirin acetylates a specific serine residue in $\mathrm{COX}-2$ rendering the enzyme inactive in terms of conversion of arachidonic acid to prostaglandins. However, acetylated COX-2 is still capable of converting arachidonic acid to 15-epi $\mathrm{LXA}_{4}$ or aspirin triggerd LX (ATL). ATL and $\mathrm{LXA}_{4}$ have similar biological actions including a range of antiinflammatory effects that include suppression of many functions of neutrophils. Like prostaglandins, $\mathrm{LXA}_{4}$ has a potent protective effect in the stomach.

\section{NITRIC OXIDE}

Nitric oxide is a ubiquitous molecule involved in a variety of biological processes. It is a signaling molecule recognized for its ability to enhance gastric mucus or alkaline secretion, inhibit gastric acid secretion and prevent neutrophil activation and adherence to vascular endothelium thus affording gastroprotection (Brzozowski et al., 2002). It is also a modulator of blood flow, gastric motility and water and electrolyte transport (Jourd'heuil et al., 1999). The importance of nitric oxide in gastrointestinal mucosal defense is well established. It is a weak radical produced from L-arginine by the enzyme nitric oxide synthase and it has a dichotomous role as both a beneficial and detrimental molecule (Kubes \& McCafferty, 2000). It has the capacity to down-regulate inflammatory responses in the gastrointestinal tract, to scavenge various free radical species, and to protect the mucosa from injury induced by topical irritants (Kubes \& Wallace, 1995).

The actions of nitric oxide overlap considerably those of prostaglandins, such as modulation of activities of immunocytes, reduction of leukocyte endothelial adhesive interactions, modulation of mucosal blood flow, reduction of epithelial permeability, and stimulation of mucus and bicarbonate secretion. NO has been proven to be a primary non-adrenergic, non-cholinergic transmitter in the gastrointestinal tract. Inhibition of nitric oxide synthase results in disturbances of gastrointestinal blood flow, motility and secretion (DeMeo et al., 2002). NO produced by nitric oxide synthase is involved in the maintenance of gastrointestinal mucosal integrity through modulation of mucosal blood flow, epithelial secretion and barrier function (Barrachina et al., 2001).

In the stomach, suppression of nitric oxide synthesis renders the mucosa more susceptible to injury whereas administration of nitric oxide donors can protect stom- 
ach from injury. This has led to the development of a series of nitric oxide-releasing anti-inflammatory drugs with a greatly reduced gastrointestinal toxicity relative to parent drugs (DeMeo et al., 2002). Nitric oxide-NSAIDs have been developed like nitroaspirin and nitroparacetamol (Martin \& Wallace, 2006). There is clear evidence for an important role of nitric oxide in mediating mucosal defense under normal conditions but the situation is much more complex when the mucosa is inflamed or injured.

Some studies suggest that nitric oxide contributes to injury whereas others suggest that it acts in a protective manner. Nitric oxide has been suggested to react with the superoxide anion produced by activated neutrophils, to form another potent oxidant, peroxynitrite. Administration of peroxynitrite into the colon produces widespread injury and inflammation. Accordingly, nitric oxide synthase inhibitors have been shown to reduce the severity of colonic damage and inflammation (DeMeo et al., 2002).

\section{POLYAMINES}

Polyamines are involved in cell migration and proliferation that result in the repair of mucosal lesions (Johnson \& McCormack, 1999). The initiation of the repair phase of inflammatory response is accompanied by an increase in polyamine synthesis. Polyamines are initially produced after conversion of arginine to ornithine by the enzyme arginase. Ornithine can then be converted to the pro-proliferative polyamines by ornithine decarboxylase. The initiation of the biosynthesis of cationic higher polyamines (putrescine, spermidine, spermine) by ornithine decarboxylase has proven to be an important stimulus for cell proliferation. An increase in intracellular polyamine levels and ornithine decarboxylase activity is associated with rapid growth rates (DeMeo et al., 2002). The mucosa of the gastrointestinal tract has one of the shortest turnover times of any tissue in the body (Naito, 2006).

The repair of damaged mucosa occurs through two mechanisms:

1. Mucosal restitution: rapid, consists of the migration of remaining viable cells from areas adjacent to the lesion to cover the denuded area. This process requires an intact lamina propria as a matrix for the cell to migrate over.

2. Actual replacement of lost cells by cell division. It depends on DNA synthesis, which begins approximately 12 hours after the start of healing.

Polyamines are required for both these processes (Johnson \& McCormack, 1999).

\section{OXIDATIVE STRESS}

Molecular oxygen is the premier biological electron acceptor indispensable in fundamental cellular functions. However, with the beneficial properties of oxygen comes the inadvertent formation of reactive oxygen species (ROS) such as superoxide, hydrogen peroxide and hydroxyl radical. If unabated, ROS pose a serious threat to or cause death of aerobic cells. To minimize the damaging effects of ROS, aerobic organisms (Scandalios, 2005) have evolved complex antioxidant mechanisms comprising low molecular weight compounds viz: glutathione and vitamin E, and enzymes such as catalase, superoxide dismutase and glutathione peroxidase (Rial \& Zardoya, 2009).
In the last one and a half decades, the concept of free radical-mediated oxidative stress (OS) has gained tremendous scientific momentum, from studying its role in the pathophysiology of disease to therapeutic implications (Bhardwaj, 2008). It has become increasingly apparent that oxidants, in addition to being agents of cytotoxicity, can play an important role in mediating specific cell responses and expression of genes involved in degenerative pathophysiologic states such as inflammation and cancer (Aw, 1999).

Oxidative stress is a state in which toxic reactive oxygen species (ROS) overcome the endogenous antioxidant defense of the host. This state results in an excess of free radicals which can react with cellular lipids, proteins and nucleic acids leading to cellular injury and eventual organ dysfunction. Gastric dysfunction is a highly complex biochemical protective response to cellular tissue injury. Helicobacter pylori infection and NSAID ingestion are major causative factors in the pathogenesis of gastric mucosal oxidative injury in humans. In response to H. pylori infection or NSAID, neutrophils are recruited to the site of inflammation and generate ROS and nitrogen reactive species (Dovhanj et al., 2010).

The major sources of reactive oxygen species are mitochondrial and cytochrome P-450 enzymes, endotoxinactivated macrophages, including Kuppfer cells cytokineactivated neutrophils and hypoxia-activated hypoxanthine-xanthine oxidase system (Naito, 2006).

Free radicals are atoms or atom groups that contain unpaired electrons. Since electrons have a very strong tendency to exist in a paired rather than unpaired state, free radicals indiscriminately pick up electrons from other atoms, converting them into secondary free radicals. Thus a chain reaction is triggered that can cause substantial biological damage. Reactive oxygen species are oxygen-derived small molecules including oxygen radicals [superoxides $\left(\mathrm{O}_{2}^{-*}\right)$, hydroxyl $\left(\mathrm{OH}^{*}\right)$, peroxyl $\left(\mathrm{RO}_{2}{ }^{\circ}\right)$ ] and certain non-radicals that are either oxidizing agents and /or are easily converted into radicals, such as hypochlorous acid ( $\mathrm{HOCl})$, ozone $\left(\mathrm{O}_{3}\right)$ and hydrogen peroxide $\left(\mathrm{H}_{2} \mathrm{O}_{2}\right)$.

The physiological generation of free radicals can occur as a byproduct of biological reactions in mitochondria, peroxisomes, or those catalyzed by cytochrome P-450. In a resting cell, superoxide ion is produced at $1-2 \%$ of daily oxygen consumption during electron transfer and oxidative phosphorylation for ATP generation by mitochondria. Mitochondrial ROS are recognized as regulators of mitochondrial functions including electron transfer chain enzymes and mitochondrial membrane potential.

Overproduction of ROS is most frequently caused by excessive stimulation of NADPH oxidase by cytokines or by dysregulation of mitochondrial electron transport chain or xanthine oxidase and results in oxidative stress. Oxidative stress can be an important mediator of damage to cell structures and consequently various disease states (Dovhanj et al., 2010).

\section{PRODUCTION OF ROS}

Reactive oxygen species produced within gastrointestinal the lumen (Gristan et al., 1987) contribute to tissue destruction. They have been implicated in various gastrointestinal abnormalities including acid-related peptic diseases (Einzhamer et al., 1995).

Conversely, when present in low amounts nitric oxide (NO), superoxide anion, and related reactive oxygen 
species play an important role as mediators in signaling processes through which, paradoxically, many ROS-mediated responses can protect cells against oxidative stress by induction of redox homeostasis (Kvietys \& Granger, 2012). Therefore, diseases associated with free radical overproduction are produced by "blazed ROS production" far beyond the host's capacity to quench.

Free radicals have been implicated in the pathogenesis of diverse gastrointestinal diseases, including reflux esophagitis, $H$. pylori associated gastritis, ulcerative colitis, NSAID-induced enteritis and associated colorectal cancer.

ROS generation is generally a cascade of reactions that starts with the production of superoxide, which rapidly dismuates to hydrogen peroxide. The ROS generation cascade includes the reaction of superoxide with nitric oxide to form peroxynitritrite, the peroxidase-catalysed formation of hypochlorous acid to form hydrogen peroxide, or the iron-catalysed Fenton reaction leading to generation of hydroxyl radical. Free radicals may act as direct or indirect damaging agents through their reaction with other components in the cell. The enzymatic and non-enzymatic antioxidant defense systems including catalase (CAT), superoxide dismutase (SOD) and glutathione peroxide (GPx) scavenge and regulate overall ROS levels to maintain physiological homeostasis. Lowering ROS levels below the homeostatic point may interrupt the physiological role of oxidants in cellular proliferation and host defense. Under certain conditions, e.g., the presence of an elevated concentration of transition metal $(\mathrm{Fe} / \mathrm{Cu})$ ions, drug metabolism or ischaemia-reperfusion, ROS generation overwhelms the cellular antioxidant defense resulting in oxidative stress.

\section{MARKERS OF OXIDATIVE STRESS}

Oxidative stress can be detected by a number of markers. These include 8-hydroxy-2-deoxyguanosine (8-OHdG), hexanoyl lysine, and isoprostane among which $8-O H d G$ is a well- known biomarker for oxidative damage in diabetes mellitus, different cancer types, and diverse gastrointestinal tract inflammatory diseases including $H$. pylori associated gastritis. 8-Hydroxy-2-deoxyguanosine is not merely a biomarker of oxidative stress but also a remedy for oxidative stress implicated in gastrointestinal diseases (Gionchetti et al. 2002),. Reactive oxygen species attack guanine base in DNA easily and form 8-OHdG. An increased level of $8-\mathrm{OHdG}$ is generally regarded as a biomarker of mutagenesis consequent to oxidative stress (Ock et al., 2012). Since oxidized guanine is membrane permeable, it can be detected in urine or serum of patients. In addition to other markers, hexanoyl lysine formed by oxidation of fatty acids and isoprostane formed when phospholipids are exposed to oxidative stress, are also excreted in urine. Even peroxidised lipids produced during oxidative stress can be detected in the serum (Kim et al., 2012).

\section{OXIDATIVE STRESS IN ESOPHAGEAL DISEASES}

Oxidative stress to esophageal mucosa plays a key role in the pathogenesis of gastroesophageal reflux disease (GERD). Barrett's esophagus is a well recognized risk of esophageal cancer. It can progress from a simple metaplastic change, through a low grade dysplasia, to high grade dysplasia and subsequently to adenocarcinoma. Clinical investigations have shown elevated ROS levels in patients with Barrett's esophagus (Olyaee et al., 1995).

\section{OXIDATIVE STRESS IN GASTRIC DISEASES}

Gastric cancer is the second leading cause of cancerrelated mortality worldwide. Infection with $H$. pylori increases the risk of developing gastric cancer more than four fold. In $H$.pylori induced gastritis, ROS are usually released from activated neutrophils. This is considered a major mechanism of $H$. pylori induced gastric mucosal injury. Inactivated neutrophils, myeloperoxidase lead to the formation of a potent oxidant, hypochlorous anion, which is formed from $\mathrm{Cl}^{-}$in the presence of $\mathrm{H}_{2} \mathrm{O}_{2}$ The hypochlorous anion reacts with ammonia produced by $H$. pylori associated urease to yield monochloramine $\left(\mathrm{NH}_{2} \mathrm{Cl}\right)$ which can freely penetrate biological membranes to oxidize intracellular components. In patients with $H$. pylori infection, tissue levels of malondialdehyde (MDA) significantly elevated, which is in turn associated with an increased turnover of glutathione. Chronic gastritis can be described as the accumulation of continuous oxidative DNA damage with mutagenic and carcinogenic potential.

Severe oxidative stress produces reactive oxygen species and induces uncontrolled lipid peroxidation. Lipid peroxidation measured as plasma thiobarbituric acid reactive substance (TBARS), is elevated significantly in stomach cancer (Reddy et al., 2010). High levels of TGF- $\beta_{1}$ and enhanced TGF- $\beta_{1}$ receptor signaling are related to the pathology of cancer. This is also caused by oxidative stress and lipid peroxide production.

Antioxidant treatment may protect the gastric mucosa from persistent pathology after eradication of $H$. pylori infection. Vitamins $\mathrm{A}, \mathrm{C}$ and $\mathrm{E}$ have been suggested to protect the gastric mucosa in $H$. pylori eradicated patients, probably due to attenuation of oxidative stress and proinflammatory cytokines (Kim et al., 2012).

\section{OXIDATIVE STRESS IN INFLAMMATORY BOWEL DISEASE}

Inflammatory bowel disease is caused by oxidative damage by free radicals accompanied by reduced antioxidant levels. It is a condition of unknown etiology characterized by persistent mucosal inflammation at different levels of the gastrointestinal tract (Gill \& Guarner, 2008). It has two manifestations, Crohn's disease and ulcerative colitis. It is a widespread and debilitating disease characterized by immune cell infiltration and immune-mediated destruction of the gastrointestinal tract. Although the causes of inflammatory bowel disease are not yet known, genetic factors certainly play some role (Verma et al., 2007). Activated neutrophils and macrophages are responsible for ROS generation and the levels of ROS can be correlated with the severity of inflammatory changes in colonic mucosa (Kim et al., 2012).

\section{APPROACHES TO REDUCE OVER INFLAMMATION AND MUCOSAL DAMAGE}

Non-steroidal anti-inflammatory drugs (NSAIDs) represent one of the most widely used classes of drugs and are primarily used to alleviate the symptoms of inflammatory disorders, however, their use is significantly limited by their ability to induce the formation of erosions and ulcers in the gastrointestinal tract. There is therefore a need for analgesic and anti-inflammatory drugs that will provide patients with symptomatic relief without causing gastrointestinal injury. 
The effectiveness of NSAIDs in reducing pain and swelling lies in their ability to inhibit prostaglandin synthesis. COX-1 and COX-2 are the key enzymes for the synthesis of prostaglandins. Prostaglandins mediate many components of gastric mucosal defense including mucus and bicarbonate secretion, mucosal blood flow, epithelial cell turnover and mucosal immunocyte function. In the healthy stomach, most of the prostaglandins are produced by COX-1. However, COX-2 activity can be rapidly induced in the stomach in response to subtle changes including the administration of aspirin or a short period of ischaemia.

\section{SELECTIVE COX-2 INHIBITORS}

NSAIDs can impair mucosal defense and render the stomach more susceptible to injury. While conventional NSAIDs inhibit both COX-1 and COX-2, selective inhibition of the COX-2 isoform was considered therapeutically more desirable, the hypothesis being that suppression of COX-2 activity would reduce the production of prostaglandins at the site of inflammation while sparing COX- 1 mediated prostaglandin synthesis in the stomach. As a result these agents might be expected to offer more favourable safety profile than non-selective NSAIDs with respect to upper gastrointestinal tract bleeding (Lanas, 2010).

NSAID-induced gastric damage requires suppression of both COX-1 and COX-2. The observation that selective inhibition of COX-2 does not result in significant damage, has led to the proposal that selective COX-2 inhibitors may be gastric sparing compounds. Clinically, selective COX-2 inhibitors produced severe gastrointestinal complications less frequently than did conventional NSAIDs. Rofecoxib and Celecoxib have been associated with a fourfold reduction in ulcers even at a high dose in comparison with normal doses of standard NSAID (Hawkey \& Langman, 2003). However, the accumulating understanding of the physiological roles of COX-2 in a variety of tissues including stomach and kidney, together with the withdrawal from the market of Rofecoxib and Valdecoxib because of cardiovascular toxicity have challenged the benefits of selective COX-2 inhibitors (Di \& Izzo, 2003).

\section{CANNABINOIDS}

$\Delta^{9}$-Tetrahydrocannabinol, the active ingredient of marijuana, as well as endogenous and synthetic cannabinoids exert many biological functions by activating two types of cannabinoid receptors, $\mathrm{CB}_{1}$ and $\mathrm{CB}_{2}$. $\mathrm{CB}_{1}$ receptors have been detected on enteric nerves and pharmacological effects of their activation include gastroprotection, reduction of gastric and intestinal motility, and reduction of intestinal secretion. Activation of these receptors can be used for the treatment of a number of gastrointestinal diseases including gastric ulcers, inflammatory bowel disease, Crohn's disease, paralytic ileus and gastroesophageal reflux disease. The most commonly used cannabinoid clinically is nabilone (Coruzzi et al., 2007).

\section{RECENT DEVELOPMENTS}

\section{Terminal prostaglandin synthase inhibitors}

The cardiovascular toxicity of selective COX-2 inhibitors is possibly a consequence of the inhibition of syn- thesis of prostacyclin $\left(\mathrm{PGI}_{2}\right)$ which has anti-thrombotic properties, while sparing the synthesis of thromboxane $\left(\mathrm{TXA}_{2}\right)$, a prothrombotic substance.

$\mathrm{PGE}_{2}$ is the prostaglandin primarily associated with inflammation. Therefore, selective inhibition of $\mathrm{PGE}_{2}$ synthesis could be a rational approach to reduce inflammation without producing the cardiovascular and gastrointestinal toxicity associated with NSAIDs. This may be achievable through one of the PGE synthase namely, mPGES-1. There is a presumption that prostaglandins other than $\mathrm{PGE}_{2}$ will be sufficient to maintain the gastrointestinal mucosal defense.

\section{Gaseous mediator releasing NSAIDs}

The hypothesis that deficiency of prostaglandins in gastrointestinal mucosa leads to ulceration has led to several new approaches to drug development involving supplementation of either prostaglandins or mediators that carry out similar functions. Earlier, prostaglandin analogs were developed for use in prophylaxis of NSAIDinduced injury; however, a high rate of side effects (e.g., diarrhea, abdominal pain) limited their widespread use. The discovery that other endogenous mediators produced many of the same effects of prostaglandins in terms of mucosal defense has led to the development of novel NSAIDs that slowly release gastroprotective substances. Nitric oxide (NO) and hydrogen sulphide $\left(\mathrm{H}_{2} \mathrm{~S}\right)$ are endogenous gastric mediators that exhibit many prostaglandin-like effects in the gastrointestinal tract. They are vasodilators and can inhibit leukocyte adherence to the vascular epithelium. Inhibition of mucosal synthesis of $\mathrm{NO}$ or $\mathrm{H}_{2} \mathrm{~S}$ renders the stomach more susceptible to the damaging effects of NSAIDs and impairs healing of existing ulcers. Because $\mathrm{NO}$ and $\mathrm{H}_{2} \mathrm{~S}$ are potent antiinflammatory agents, there is the possibility of boosting the anti-inflammatory activity of NSAIDs by coupling NSAIDs with these gaseous mediators. In the case of NO-NSAIDs there is a substantial body of evidence that these drugs produce less gastrointestinal injury than the parent NSAIDs both in animals and clinical trials (Wallace \& Vong, 2008).

\section{Association of phosphatidylcholine and NSAIDs}

This is a novel strategy used to reduce gastrointestinal toxicity. Novel NSAID products utilize a lipid based formulation containing soy lecithin enriched in phosphatidylcholine (PC) in a neutral lipid or oil matrix which is similar to a self-emulsifying drug delivery system. Phosphatidylcholine is a functional excipient, acts as a solubilizing agent by forming a non-covalent with NSAIDs. It brings out reversible changes in biophysical characteristics of NSAIDs. This phosphatidylcholine-NSAID complex is more lipophilic. The enhanced lipid solubility of the drug promotes its transit across the hydrophobic mucus layer of the upper gastrointestinal tract and presumably also of the stomach with reduced mucosal injury. The phosphatidylcholine oil neither impedes the bioavailability nor changes the pharmacological activity of the active ingredient (Lichtenberger et al., 2009).

\section{Role of probiotics in prevention and treatment of gastrointestinal inflammation}

The human gastrointestinal tract is home to diverse and vast communities of microorganisms representing over 400 cultivable species (Gill \& Guarner et al., 2004). Probiotics are live microorganisms administered to alter the intestinal microflora and confer a beneficial effect on 


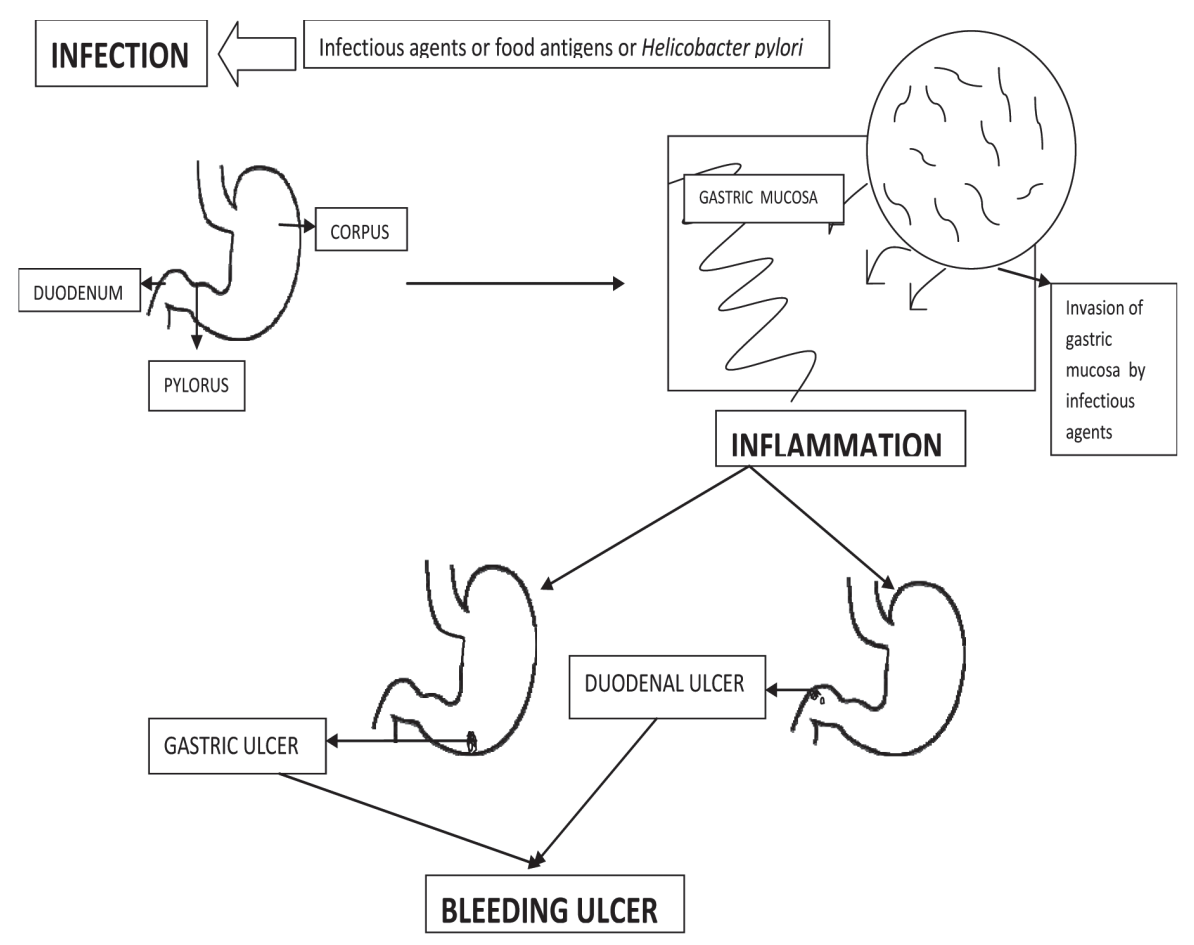

Figure 1. Schematic representation of ulcer formation.

health (Anodh \& Fujiyama, 2006). Most probiotics fall into the group of organisms known as lactic acid producing bacteria and are normally consumed in the form of yogurt, fermented milk or other fermented foods (Parvez et al., 2006). Recent evidence have suggests a potential therapeutic role of probiotics in the prevention or treatment of gastrointestinal diseases (Bizello et al., 2002). In the last few years they became increasingly popular as nutritional supplements especially to achieve reduction of gastrointestinal complaints and common infectious illnesses (Lamprecht et al., 2012). Clinical studies have established that certain probiotics are useful in a variety of intestinal disorders including viral diarrhea, antibiotic associated diarrhea, and traveller's diarrhea (Vanderhoof et al., 2001). They have been utilized in an attempt to reestablish the balance of the host microflora and attenuate aberrant response (Culligan et al., 2009).

Probiotic therapy is based on the concept of normal, healthy microflora. Components of the human intestinal microflora or organisms entering the intestine may have harmful or beneficial effects on human health. Abundant evidence implies that specific strains selected from the healthy gut microflora exhibit powerful antipathogenic and anti-inflammatory capabilities (Isolauri et al., 2002).

Probiotics show beneficial health effects if ingested in sufficient number. The bacteria most commonly associated with probiotic activity are lactobacilli, bifidobacteria, and streptococci, but other non-pathogenic bacteria such as some strains of Escherichia coli, and non-bacterial organisms such as the yeast Saccharomyces boulardii have been used as well. For clinical application probiotic strains must be both bile-and acid-resistant and must retain metabolic activity within the intestinal lumen where ideally, they should survive but not persist in the long term.

Various mechanisms have been proposed for the action of probiotics:

1. Antagonistic activity against pathogenic bacteria either by inhibition of adherence or translocation or by production of anti-bacterial substances such as antimicrobial peptides and hydrogen peroxide.

2. Production of nutrients of special importance to the intestine such as short chain fatty acids and vitamins (Kubes et al., 2000).

Oral introduction of probiotics has been shown to reinforce the various lines of gut defense: immune elimination, immune exclusion, and immune regulation. Probiotics also stimulate nonspecific host resistance to microbial pathogens and thereby aid in their eradication (Baarachina et al., 2001).

\section{Inflammation and Cancer}

The association between inflammation and cancer has long been appreciated (Nahoum, 2007). Infection and chronic inflammation contribute to about $25 \%$ of all cancers. Patients with chronic inflammatory and oxyradical overload (oxidative stress) conditions are at a much higher risk of developing cancer. A number of mediators of inflammatory response, viz. cytokines, free radicals, prostaglandins and growth factors induce various genetic and epigenetic changes leading to alterations in critical pathways responsible for maintaining the normal homeostasis and ultimately leading to the development and progression of cancer (Hussain \& Harris., 2007).

A well-known example is that chronic ulcerative colitis may lead to colorectal cancer. Ulcerative colitis is a chronic inflammatory bowel disease associated with multiple colonic (chronic, recurrent ulcers and inflammation of colonic mucosa) (Tolstanova et al., 2012) and extraintestinal complications. The most severe of these is the development of colorectal cancer. The incidence of colorectal cancer in ulcerative colitis is approximately 4/1000 patients. Chronic inflammation leads to progressive dysplasia and eventually adenocarcinoma (Sunkara et al., 2011). Ulcerative related colorectal cancer is responsible for less than $2 \%$ of all colorectal cancers (Triantafillidis et al., 2009). 
An increased production of mucosal proinflammatory cytokines or other inflammatory mediators such as reactive oxygen species and prostaglandins results in damage to DNA, RNA, proteins or lipids. They induce formation of adducts to DNA, generate point mutations in genes like the tumor suppressor gene TP53, which is mutated early in ulcerative colitis inflamed mucosa. Increase in the local concentration of proinflammatory cytokines and prostaglandins leads to inhibition of apoptosis, favors cell proliferation and thus promotes carcinogenesis (Reimund et al., 2012)

\section{REFERENCES}

Anodh A, Fujiyama Y (2006) Therapeutic approaches targetting intestinal microflora in Inflammatory Bowel Disease. World J Gastroentero 12: 4452-4460.

Aw TY (1999) Molecular and cellular responses to oxidative stress and changes in oxidation-reduction imbalance in the intestine. Am J Clin Nutr 70: 557-565.

Baarachina, Esplugues JV, Panes J (2001) Role of nitric oxide in gastrointestinal inflammation and ulcerative diseases: perspective for drugs development. Curr pharmdesign 7: 31-48.

Bhardwaj P (2008) Oxidative stress and antioxidants in gastrointestinal diseases. Trop Gastroenterol 29: 129-135.

Bonnans C, Vachier I, Chavis C, Goodard P, Bousquet J, Chanez P (2002) Lipoxins are potential endogenous anti-inflammatory mediators in asthma. Am J Resp Crit Care 165: 1531-1535.

Brenchley JM, Douek DC (2012) Microbial translocation across GI tract. Annu Rev Immunol 30: 149-173.

Brzozowski T, Konturek PC, Pajdo R, Ptak BA, Kwiecien S, Pawlik M, Drozdowicz D, Silwowski Z, Brzozowski B, Konturek SJ, Pawlik WW (2008) Physiological mediators in non Steroidal anti-inflammatory drugs (NSAIDs) induced impairment of gastric mucosal defense and adaptation-focus on nitric oxide and lipoxins. $J$ Physiol Pharmacol 59: 89-102.

Chaitanya GV, Franks SE, Cromer W, Wells SR, Beinkowska M, Jennings MH, Ruddel A, Ando T, Wang Y, Gu Y, Sapp M, Mathis JM, Jordan PA, Minagar A, Alexander JS (2010) Differntial cytokine responses in human and mouse lymphatic endothelial cells to cytokines in vitro. Lymphatic Res Biol 8: 155-164.

Coruzzi G, Venturi N, Spaggiari S (2007) Gastrointestinal safety of novel non steroidal anti- inflammatory drugs: selective COX-2 inhibitors and beyond. Acta Biomed 78: 96-110.

Culligan EP, Hill C, Sleator RD (2009) Probiotics and gastrointestinal diseases, successes, problems and future prospects. Gut Pathog 23: $1-19$.

DeMeo MT, Mutlu EA, Keshavarzian A, Mark T, Tobin MC (2002) Intestinal permeation and gastrointestinal Disease. J Clin Gastroenterol 34: 385-396.

Di CG, Izzo AA (2003) Cannabinoids for gastrointestinal diseases: potential therapeutic applications. Expert Opin Inv Drug 12: 39-49.

Dovhanj J, Svagelj Drazen (2011) Oxidative stress pathway driven by inflammation in gastric mucosa. Gastritis and Gastric Cancer New Insights in Gastroprotection Diagnosis and Treatment ISBN: 978-953-307375-0: 47-62.

Gill HS, Guarner F (2004) Probiotics and human health: a clinical perspective. Postgrad Med J 80: 516-526.

Gionchetti P, Rizzello F, Campieri M (2002) Probiotics in gastroenterology. Curr Opin Gastrorn 18: 235-239.

Grisham MB, Von RC, Smith BF, Lamont JT, Granger DN (1987) Interaction between oxygen radicals and gastric mucin. Am I Physiol 253: G93-G96.

Guarner F (2007) Prebiotics in inflammatory bowel disease. Br J Nutr 1: S85-S89.

Hawkey CJ, Langman CJ (2003) Non steroidal anti-inflammatory drugs: overall risks and management- complementary roles for COX-2 inhibitors and proton pump inhibitors. Gut 52: 600-608.

Hussain SP, Harris CC (2007) Inflammation and cancer: an ancient link with novel potentials. Int J. Cancer 121: 2373-2380.

Isolauri E, Kirjavainen P, Salminen S (2002) Probiotics: a role in treatment of intestinal infection and inflammation. Gut 50: iii54-iii59.

Johnson LR, McCormack SA (1999) Healing of gastrointestinal mucosa: involvement of polyamines. News Physiol Sci 14: 12-17.

Jourd'heuil D, Grisham MB, Granger DN (1999) Nitric oxide and gut. Curr Gastroenterol Rep 1: 384-388.

Kim YJ, Kim EH, Hahm KB (2012) Oxidative stress in inflammation based gastrointestinal tract diseases. J Gastroen Hepatol 27: 1004 1010.

Kubes P, McCafferty DM (2000) Nitric oxide and intestinal inflammation. Am J Med 109: 150-158.
Kubes P, Wallace J L (1995) Nitric oxide as mediator of gastrointestinal mucosal injury?-Say it ain't so. Mediat Inflamm 4: 397-405.

Kvietys PR., Granger DN (2012) Role of reactive oxygen and nitrogen species in the vascular responses to inflammation. Free Radical Bio Med 52: 556-592.

Lamprecht M, Bogner S, Schippinger G, Steinbauer K, Fankhauser F, Hallstroem S, Schuetz B, Gerilberger JF (2012) Probiotic supplementation affects markers of intestinal barrier, oxidation and inflammation in trained men, a randomized double blinded, placebo controlled trial. J. Internat Soc Sports Nutr 9: 45-58.

Lanas A (2010) A review of gastrointestinal safety data- a gastroenterologist's perspective. Rheumatology 49: ii3-ii10.

Lichtenberger LM, Barron M, Marathi U (2009) Association of phosphatidylcholine and NSAIDs as a novel strategy to reduce gastrointestinal toxicity. Drugs Today 45: 877-890.

Macdonald LJ, Boody SC, Denison FC, Sales KJ, Jabbour HN (2011) A role for lipoxin $\mathrm{A}_{4}$ as an anti-inflammatory mediator in the human endometrium. Reproduction 142: 345-352.

Martin GR, Wallace JL (2006) Gastrointestinal inflammation: a central component of mucosal defense and repair. Exp Biol Med 231: 130-137.

Medilexicon's Medical Dictionary.

Nahoum SR (2006) Why cancer and inflammation. Yale J Biol Med 79: $123-130$.

Naito Y (2006) Oxidative stress in digestive diseases. I Clin Biochem Nutr 39: 55.

Neerudu M, Rajababu, Reddy H, Koppolu P (2009) Antiinflammatory mediators. IJDA 2: 182-184.

Ock CY, Kim EH, Choi DJ, Lee HJ, Hahm KB, Chung MH (2012) 8-Hydroxyguanosine: not mere biomarker for oxidative stress, but remedy for oxidative stress implicated gastrointestinal diseases. World J Gastroentero 18: 302-308.

Olyaee M, Sontag S, Salman W, Schnell T, Mobarhan S, Eiznhamer D, Keshavarzian A (1995) Mucosal Reactive Oxygen Species in oesophagitis and barrett's oesophagus. Gut 37: 168-173.

Parvez S, Malik KA, Ah KS, Kim HY (2006) Probiotics and their fermented food products are beneficial for health. I Appl Microbiol 100: 1171-1185.

Pathmakanthan S, Hawkey C (2000) A lay doctor's guide to inflammatory process in the gastrointestinal tract. Postgrad Med 76: 611-617.

Rea WJ, Patel K (2010) Regulating mechanisms of chemical sensitivity. Reversibility of Chronic Degenerative Disease and Hypersensitivity 1: $337-417$.

Reddy EP, Reddy VS, Chandra MK, Rao PVLNS (2010) Physiological antioxidant system and oxidative stress in stomach cancer patients with normal renal and hepatic function. Online J Health Allied Scs 8: 9-12.

Reimund JM, Tavernier M, Veinnot S, Hussein IAH, Dupont B, Justum AM, Jurjus AR, Freund JN, Lecheverel M (2012) Ulcerative colitis associated colorectal prevention by 5 -aminosalicylates: current status and perspectives. Ulcerative Colitis from Genetics to Complications 149-166.

Rial E, Zardoya R (2009) Oxidative stress, thermogenesis and evolution of uncoupling proteins. J Biol 8: 58-58.5.

Ricciotti E, Fitz GGA (2011) Prostaglandins and inflammation. Arterioscl Throm Vasc Biol 31: 986-1000.

Scandalios JG (2005) Oxidative stress: molecular perception and transduction of signals triggering antioxidant gene defenses. Braz J Med Biol Res 38: 995-1014.

Stankov SV (2012) Definition of inflammation, causes of inflammation and possible anti-inflammatory strategies Open Inflammation J 5: 1-9.

Sunkara S, Swanson G, Forsyth CB, Keshavarzian A (2011) Chronic inflammation and malignancy in ulcerative colitis. Ulcers 2011: 1-9.

Tolstanova G, Deng X, French SW, Lungo W, Paunovic B, Khomenko T, Ahluwalia A, Kalpan T, Dacosta IM, Tarnawski A, Szabo S, Sandor Z (2012) Early endothelial damage and increased colonic vascular permeability in the development of experimental ulcerative colitis in rats and mice. Lab Invest 92: 9-21.

Triantafillidis JK, Nasioulas G, Kosmidis PA (2009) Colorectal cancer and inflammatory bowel disease: epidemiology, risk factors, mechanisms of carcinogenesis and prevention strategies. Anticancer Res 29: $2727-2737$.

Vanderhoof JA, Young RJ (2001) The role of probiotics in treatment of intestinal infections and inflammation. Curr Opin Gastroenterol 17: 58-62.

Verma AK, Verma R, Paul J (2007) Role of gut flora in inflammatory bowel disease - a state of art. Communicating Current Research and Educational Topics and Trends in Applied Microbiology 705-718.

Wallace JL, Chin BC (1997) Inflammatory mediators in gastrointestinal defense and injury. Proc Soc Exp Biol Med 214: 192-203.

Wallace JL, Granger DN (1996) The cellular and molecular basis of gastric mucosal defense. FASEBJ 10: 731-740.

Wallace JL, Vong L (2008) NSAID induced gastrointestinal damage and the design of GI sparing NSAIDs. Curr Opin Inv Drug 9: 1151-1156. 